\title{
Contraste experimental de un modelo probalístico para el dimensionamiento de elementos de vidrio plano con consideraciones de modo mixto de carga
}

\author{
Ma.A. GARCíA PRIETO, A. MIRANDA PRIETO, A. BERNARDO SÁNCHEZ, Ma.j. LAMELA REY, A. FERNÁNDEZ CANTELI \\ Departamento de Construcción e Ingeniería de Fabricación. E.T.S. de Ingenieros Industriales de Gijón. Universidad de Oviedo
}

\begin{abstract}
Una de las características del vidrio es su fragilidad y la gran dispersión de sus valores de resistencia mecánica, motivado fundamentalmente por la presencia de defectos procedentes de su fabricación o de su manipulación. Como consecuencia, las investigaciones en este material se han dirigido preferentemente a la mejora de los procesos de fabricación y al establecimiento de metodologías de diseño basadas en modelos probabilísticos.

Tradicionalmente, en el análisis de la rotura se ha despreciado la influencia del modo II o se ha representado como un porcentaje del modo I. Recientes estudios demuestran la aparición de un nuevo campo de tensiones de compresión cuando una fisura crece bajo una carga en modo II puro situado en el codo que se forma entre la fisura original y su ramificación. Este campo de compresión influye en el equilibrio energético y, en consecuencia, en la predicción de la rotura.

En colaboración con el CIDA de Cristalería Española, S.A. se ha desarrollado un procedimiento para el dimensionamiento probabilista de elementos de vidrio plano para edificación. En este artículo se propone ahora una mejora del modelo, considerando la existencia de un modo mixto de carga y procediendo posteriormente a su contraste experimental.
\end{abstract}

Palabras clave: Modo mixto, modelo probabilístico, vidrio plano, mecánica de fractura

Experimental contrast of a probabilistic model to design plates of glass considering mixed mode loading

A characteristic of the glass is its fragility and the scatter of the experimental mechanical strength due to the presence of defects that appear in the process of manufacturing or handling. Accordingly, investigations on this material were priorly conducted to improve the processes of manufacturing and to establish design methodologies based on probabilistic approaches.

The influence of mode II of loading was traditionally ignored or considered as ratio of mode II. Recent studies have shown that when a shear-loaded crack becomes unstable a new compressive stress concentration field builds up in the notch formed between the original crack and the branch crack. This stress field affects the energy balance and also the beginning of crack extension.

A procedure to design glass elements was developed jointly with CIDA of Cristalería Española,S.A. The aim of this work is to improve the model by the consideration of a mixed mode loading and its experimental contrast.

Keywords: Mixed mode loading, probabilistic approach, glass plates, fracture mechanics

\section{INTRODUCCIÓN}

El área de Mecánica de Medios Continuos y Teoría de Estructuras de la Universidad de Oviedo viene colaborando desde hace varios años con Cristalería Española, S.A. en diversos proyectos de investigación relacionados con acristalamientos en edificación. En este campo se ha elaborado un modelo probabilístico para el dimensionamiento de elementos de vidrio plano, basado en la teoría estadística de extremos y en consideraciones de la Mecánica de Fractura que permite una mejor comprensión del dimensionamiento a rotura y la consecuente posibilidad de consideración de márgenes de seguridad más ajustados y/o exigentes que los recomendados por el cálculo tradicional.

Actualmente se pretende desarrollar un modelo probabilístico aplicable en el dimensionamiento y la optimización en el diseño y fabricación del vidrio laminar para la industria del automóvil, concretamente de parabrisas.

Como paso intermedio en esta línea, el presente trabajo describe, en primer lugar, el modelo de dimensionamiento de placas. Dentro de este modelo se presta especial atención al criterio de rotura empleado, proponiendo finalmente un nuevo criterio que describe la rotura de una forma más fiel.

En la última parte del trabajo se comparan los resultados del modelo modificado con los obtenidos según el modelo anterior y con los correspondientes a una serie de ensayos experimentales.

\section{MODELO DE DIMENSIONAMIENTO PARA PLA-} CAS DE VIDRIO

Los elementos de vidrio en edificación son placas rectangulares de espesor constante que se calculan para soportar una carga equivalente de viento repartida uniformemente en toda la superficie.

El modelo desarrollado para el dimensionamiento de estos elementos se representa esquemáticamente en la figura 1.

A pesar de la sencillez geométrica de las placas de acrista- 
lamiento, el cálculo del estado tensional es complejo, debido al comportamiento marcadamente no-lineal de las placas de vidrio, incluso para cargas moderadas, relativamente alejadas de la de rotura.

El modelo matemático está basado en la ecuación de von Kármán [1], aplicable a placas con grandes deformaciones. Para su resolución se empleó el método de Gälerkin y el de diferencias finitas, en razón de la sencillez geométrica del contorno [1].

\subsection{Caracterización a fractura}

La tenacidad a fractura del material se determinó mediante el ensayo de indentación [2], [3]. Se trata de un ensayo habitual en este tipo de materiales, que presenta las ventajas de ser fácil de ejecutar, rápido y económico.

El valor de la tenacidad a fractura, $\mathrm{K}_{\mathrm{I} c^{\prime}}$ se calcula a partir de la medida de las huellas y fisuras originadas por un penetrador Vickers en el material, mediante la expresión:

$$
\mathrm{K}_{\mathrm{Ic}}=0,016\left(\frac{E}{H}\right)^{1 / 2}\left(\frac{P}{c^{3 / 2}}\right)
$$

En ella E representa el módulo de elasticidad del material, $\mathrm{P}$ la carga aplicada en el ensayo, $\mathrm{H}$ la dureza Vickers del material y c el radio del semicírculo que forma la fisura inducida por la indentación.

Un inconveniente de este ensayo es que la expresión (1) no se ajusta a todos los materiales, por lo que algunos autores [4] recomiendan sustituirla por la expresión (2):

$$
\mathrm{K}_{\mathrm{Ic}}=\chi \cdot\left(\frac{P}{c^{3 / 2}}\right)
$$

en la cual la constante $\chi$ se obtendría para el material en estudio, mediante el contraste del método de indentación con otro que proporcione resultados más estables.

Como método de contraste se propone la realización de ensayos de flexión a cuatro puntos sobre probetas previamente indentadas.

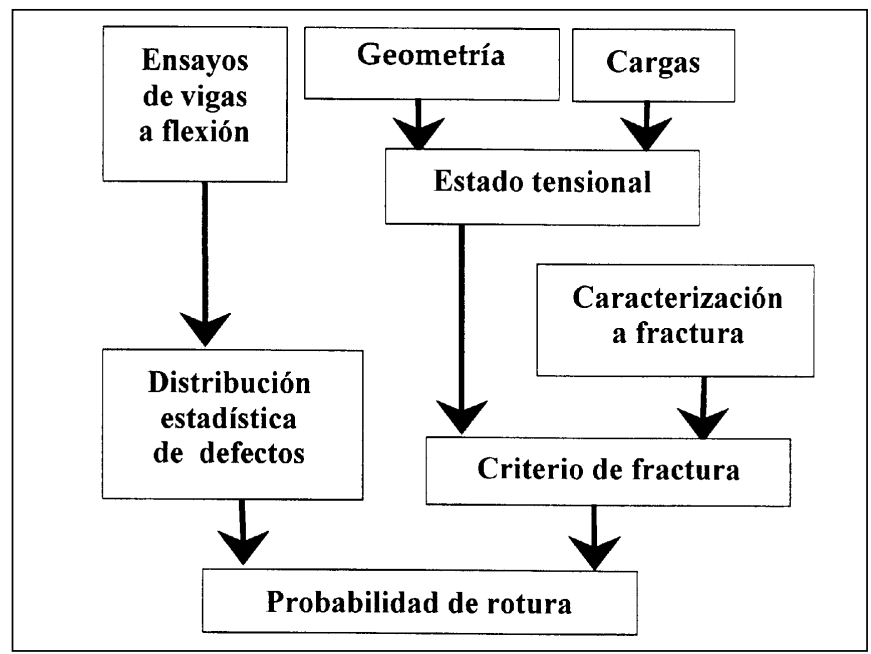

Fig. 1. Esquema del modelo para dimensionamiento de placas de vidrio.

\subsection{Criterio de rotura}

El criterio de fractura considerado es el de la energía coplanar de deformación, dado por la expresión:

$$
\left(\frac{K_{I}}{K_{I c}}\right)^{2}+\left(\frac{\alpha \cdot K_{I I}}{K_{I c}}\right)^{2}=1
$$

Para el parámetro $\alpha$, que representa la relación existente entre las tenacidades en modo I y II, se adoptó el valor 1,25 $\left(\mathrm{K}_{\mathrm{Ic}}=1,25 . \mathrm{K}_{\mathrm{IIc}}\right)$ [5]. $\mathrm{K}_{\mathrm{I}}$ y $\mathrm{K}_{\mathrm{II}}$ son los factores de intensidad de tensiones correspondientes a los modos I y II de rotura.

Se trata de un criterio comúnmente aceptado, que está basado en el establecimiento de un balance energético para una fisura cargada en modo mixto (Fig. 2).

\subsection{Defectos}

En los materiales frágiles, como el vidrio, es comúnmente aceptado que el origen de la rotura está relacionado con los defectos inherentes al proceso de fabricación y a la manipulación del material. Son muchas las imperfecciones que pueden aparecer en el vidrio en las diferentes fases, desde su fabricación: fusión, conformación y recocido, hasta su almacenamiento y puesta en servicio [6]. De ellas, algunas no tienen una apreciable influencia en la rotura, tal como es el caso de las inclusiones [7]; otras se detectan a simple vista durante el proceso de control de calidad de la fábrica, siendo el elemento defectuoso rechazado.

Con el fin de llegar a obtener un tamaño de defecto crítico, a partir del criterio de rotura, se hace necesario modelizar los defectos presentes en el material que pueden conducir a la rotura del mismo.

De acuerdo con lo anterior, en el modelo desarrollado sólo se han considerado, como defectos responsables de la rotura, las microfisuras superficiales que aparecen fundamentalmente en la fase de conformación y manufactura, debido al contacto del vidrio con los rodillos de la cadena de transporte y al proceso térmico asociado con esta operación. Los defectos considerados como responsables de la rotura se han modelizado como microfisuras semicirculares superficiales, puesto que, aunque en principio podrían asociarse simplificadamente a formas semielípticas, se demuestra, teórica y experimentalmente, que en su fase de crecimiento tienden a adoptar una forma semicircular.

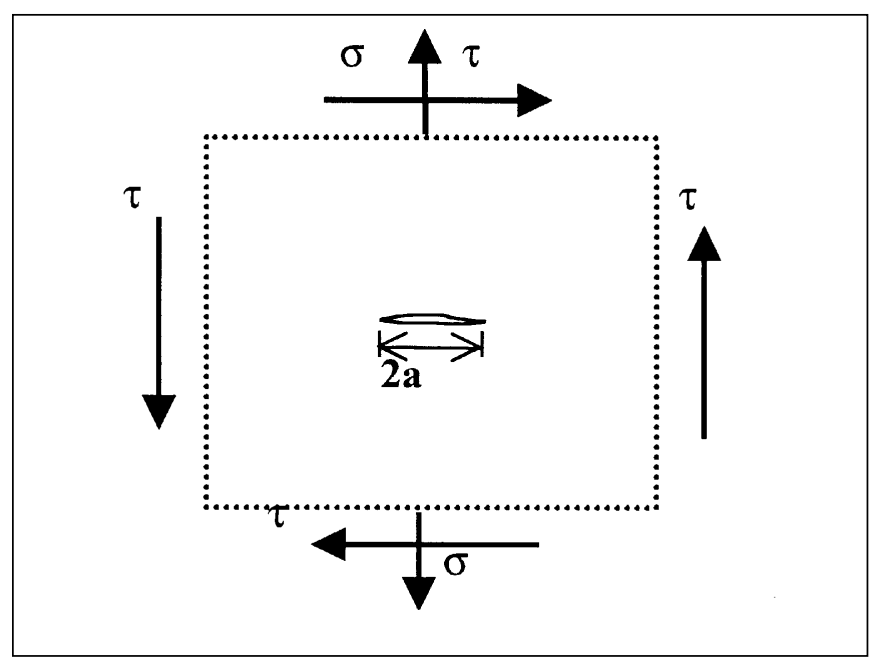

Fig. 2. Placa infinita cargada de modo mixto. 
Los defectos de borde, producidos durante los procesos de corte, manufactura, almacenamiento y manipulación, resultan igualmente significativos de cara a la rotura, $y$, aunque hasta el momento no han sido tenidos en cuenta en el modelo, en la actualidad se estudia su consideración.

Para deducir la función de distribución de los defectos en el material estudiado, se realizaron ensayos de flexión a tres puntos (Figura 3).

La tensión de rotura se calculó, tras la observación de la localización de la fractura, de acuerdo con la expresión de resistencia de materiales:

$$
\sigma_{\text {rot }}=\frac{3 \cdot P_{r o t} \cdot x}{w \cdot t^{2}}
$$

Para el tratamiento de los resultados se supuso que las roturas tenían lugar siempre bajo modo I, por lo que la función de distribución de defectos es, en realidad, la de tamaños máximos del defecto proyectado (5).

$$
\mathrm{a}_{\mathrm{rot}}=\frac{\left(K_{I c} \cdot w \cdot t^{2}\right)^{2}}{9 \cdot \pi \cdot P_{r o t}^{2} \cdot L^{2} \cdot f_{1}^{2}}
$$

En (5) $f_{1}$ representa el factor que recoge la influencia de la geometría del defecto en la rotura, w el ancho y t el espesor de la probeta ensayada.

Cabe aquí señalar que en la actualidad se recomienda realizar ensayos de flexión a cuatro puntos, debido a que la distribución de tensiones, constante en el tercio central permite una definición más exacta y fiable del área de referencia $\mathrm{A}_{\mathrm{o}^{\prime}}$ asociada a la función de distribución y consecuentemente la obtención de una f.d.d. de defectos más ajustada a la realidad.

Mediante el programa Extremes [8], basado en la Teoría Estadística de Valores Extremos, se procedió a estimar la f.d.d. de defectos superficiales máximos en el vidrio, a partir de los resultados de los ensayos de flexión realizados, comprobándose que el tamaño máximo de los defectos superficiales en este caso, sigue una ley de Gumbel para máximos.

Con el fin de tener en cuenta la influencia de la orientación de laminación se cortaron las probetas, a partir del tráver,

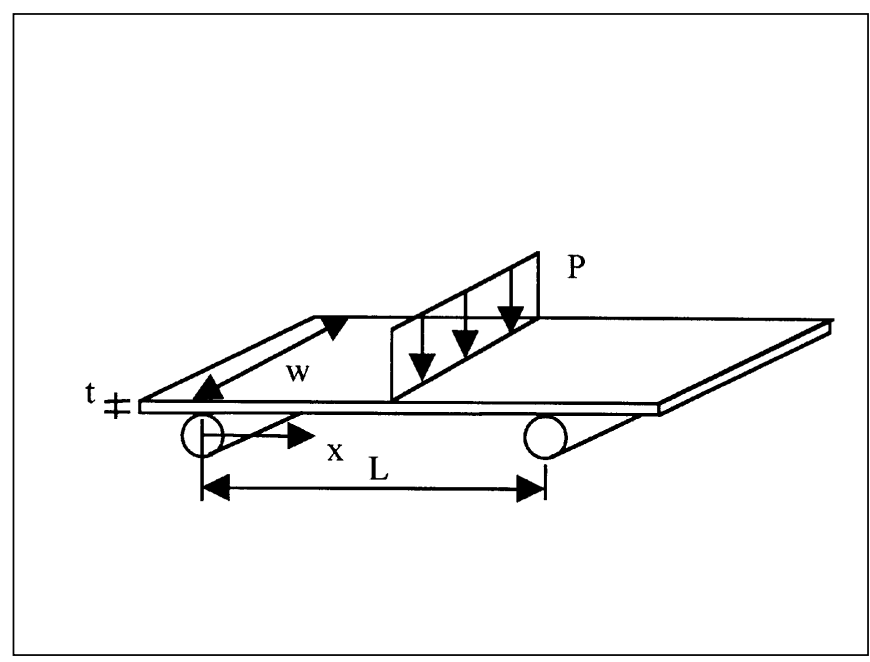

Fig. 3. Ensayo de flexión a tres puntos. para diferentes ángulos $\theta_{\mathrm{i}}$, respecto a la dirección de laminación. Por tanto, la f.d.d. de defectos asociada al área de referencia ensayada, $A_{o}$ en la figura 3, será (6):

$$
\mathrm{G}_{\theta \mathrm{i}}(\mathrm{a})=\exp \left\{-\exp \left(\frac{\lambda_{i}-a}{\delta_{i}}\right)\right\}
$$

En la que $0<\mathrm{a}<\infty, \delta_{\mathrm{i}}>0$ es el parámetro de escala y $\lambda_{\mathrm{i}}$ es el parámetro de localización para cada orientación $\theta_{\mathrm{i}}$.

Los parámetros fueron determinados con el programa Extremes [8] a partir de los resultados experimentales. La f.d.d. de defectos máximos aplicable al área elemental $\Delta x . \Delta y$, asociada al estado tensional, $\mathrm{F}_{\theta \mathrm{i}}(\mathrm{a})$, se deduce a partir de la f.d.d. de defectos máximos, $\mathrm{G}_{\theta \mathrm{i}}(\mathrm{a})$, correspondiente al área de referencia $A_{o^{\prime}}$ de acuerdo con la ley del eslabón más débil (efecto de escala):

$$
\mathrm{F}_{\theta \mathrm{i}}(\mathrm{a})=\left[\mathrm{G}_{\theta \mathrm{i}}(\mathrm{a})\right]^{\Delta \mathrm{x} \cdot \Delta \mathrm{y} / \mathrm{Ao}}
$$

\subsection{Planteamiento estadístico}

El tamaño de defecto con una orientación $\theta_{\mathrm{i}}$ determinada, necesario para que se produzca la rotura en un punto de la placa, con coordenadas $(\mathrm{x}, \mathrm{y})$, sometido a un estado tensional $\left(\sigma_{n}, \tau\right)$ debido a una carga de viento vendrá dado por (8) [2], donde $\mathrm{f}_{1} \mathrm{y} \mathrm{f}_{2}$ representan los factores geométricos de los factores de intensidad de tensiones en modo I y II, respectivamente, y on y $\tau$ las tensiones normal y tangencial que generan dichos modos de solicitación.

$$
a_{\theta_{i}}^{*}=\frac{K_{I c}^{2}}{\pi\left[f_{1}^{2} \cdot \sigma_{n}^{2}+\frac{f_{2}^{2} \cdot \tau^{2}}{0,64}\right]}
$$

El cálculo de la probabilidad de rotura de una placa dividi-

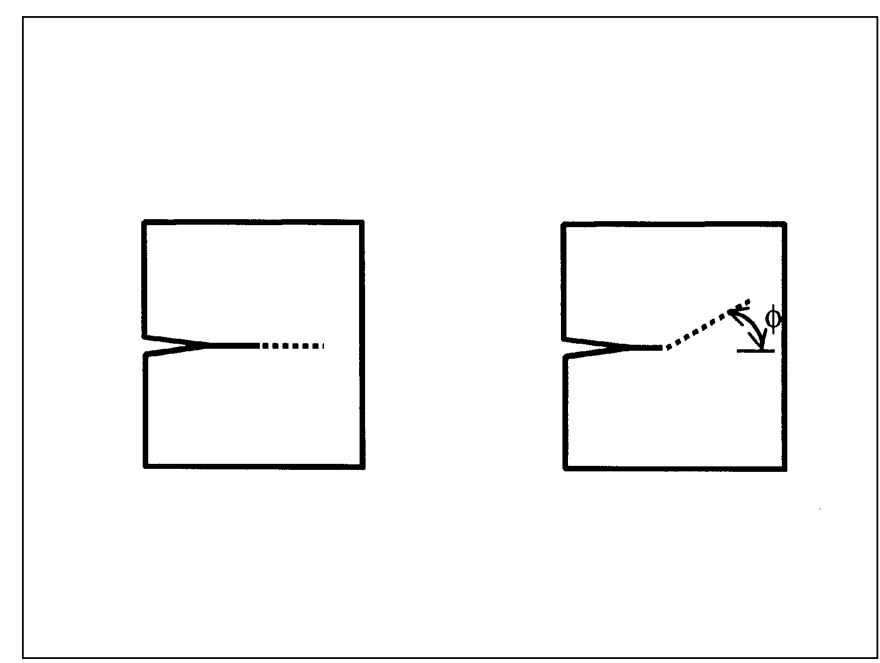

Fig. 4. Crecimiento coplanar y crecimiento no coplanar de una fisura. 
da en ' $m$ ' celdas de igual superficie se calcula mediante integración:

$$
\mathrm{P}_{\mathrm{f}}(\text { placa })=1-\prod_{i=1}^{m} P_{s}(\text { celda } i)=1-\int_{0}^{\pi} \int_{0}^{a^{*}} p(a, \theta) \cdot d a \cdot d \theta
$$

en la que, $p(a, \theta)$ representa la función de densidad bidimensional, cuyas variables son el tamaño de la fisura, a, y la orientación de la misma, $\theta$. Esta función de densidad, $\mathrm{p}(\mathrm{a}, \theta)$, se conoce de la estimación de la distribución marginal de las orientaciones, $\mathrm{p}(\theta)$ y de la función de densidad de tamaños condicionada a las orientaciones, que es la que se obtiene de los ensayos, $\mathrm{p}_{\theta}(\mathrm{a})$.

En principio, debido al acoplamiento existente entre tamaño y orientación de los defectos, no parece posible deducir esta función de distribución marginal, y en consecuencia, tampoco la distribución de densidad global bidimensional. Sin embargo, sí es factible para cada orientación, la deducción de la función de distribución de defectos 'proyectados equivalentes', a partir de resultados experimentales como los descritos anteriormente, con la cual se puede realizar ahora el cálculo bajo el supuesto de equivalencia, con el modelo real riguroso.

\section{REVISIÓN DEL CRITERIO DE FRACTURA UTILIZADO}

\subsection{Criterio anterior}

El criterio de rotura empleado en el modelo probabilístico descrito adolece de una serie de inconsistencias que es preciso corregir en lo posible, a fin de describir más fielmente el comportamiento del material en las condiciones reales de rotura bajo modo mixto (I+II), garantizando así una mayor fiabilidad en el dimensionamiento.

En primer lugar, este criterio considera un crecimiento coplanar de la fisura, lo que supone que la disposición entre la grieta y la carga actuante no experimenta variación desde la iniciación hasta la rotura. Sin embargo, está probado teórica y experimentalmente $[9,10]$ que bajo solicitación combinada (modo mixto I-II), una fisura con una determinada orientación experimenta un crecimiento según una dirección, en principio, no coincidente con la de la fisura inicial, formando un ángulo $\phi_{c}$ con ésta (Fig. 4).

En segundo lugar, en este criterio, aplicado a la rotura de placas no se plantea interacción alguna entre los factores de intensidad de tensiones de ambos modos, ni aparece depen-

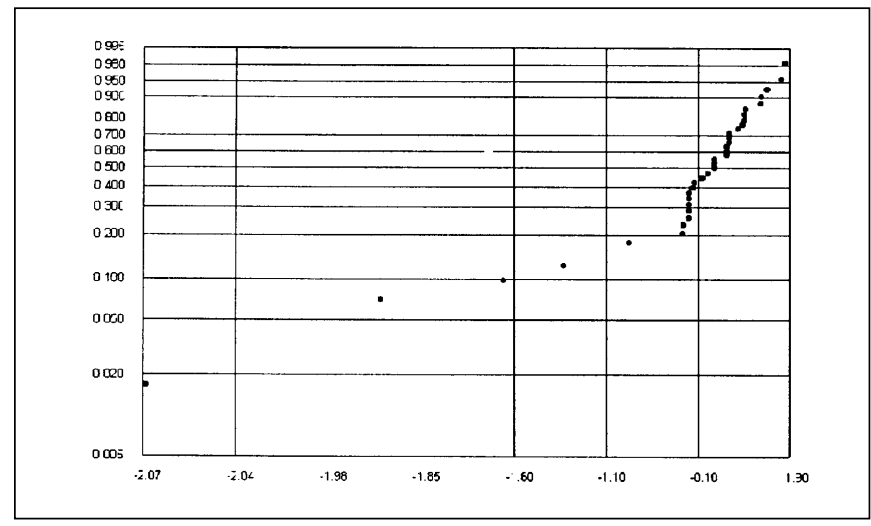

Fig. 5. Cargas de rotura normalizadas en papel de Weibull para mínimos.

dencia de los mismos respecto de las dos componentes de la tensión, $\left(\sigma_{n^{\prime}} \tau\right)$. Es decir, $\mathrm{K}_{\mathrm{I}}$ sólo dependería de $\sigma_{\mathrm{n}}$ y $\mathrm{K}_{\mathrm{II}}$ sólo de $\tau$.

Podleschny y Kalthoff $[9,10]$ han comprobado experimentalmente, que en el caso de rotura inestable bajo modo II, en el entorno de la grieta aparecen tres campos de concentración de tensiones (ver figura 4): Uno en el extremo de la grieta original, antes de la inestabilidad, otro presente en el frente de grieta de la ramificación, y un tercero, que corresponde a un campo de tensiones de compresión que se forma tras la inestabilidad en el acodamiento entre la fisura original y la ramificación. De ahí que en el establecimiento del balance energético, el criterio buscado deberá considerar la aparición del campo de tensiones de compresión en el codo, puesto que parte de esta energía elástica, supuestamente disponible para un crecimiento de la fisura, se ha de destinar a la construcción del nuevo campo de tensión.

\subsection{Nuevo criterio de rotura}

El nuevo criterio de rotura en vidrio, basado en el modelo anterior incorpora las mejoras comentadas anteriormente.

La posibilidad de crecimiento no coplanar queda recogida mediante la definición de nuevos factores de intensidad de tensiones, $\mathrm{K}_{\mathrm{MMI}}, \mathrm{K}_{\mathrm{MMI}}$ :

$$
\begin{aligned}
& K_{M M I}=\frac{1}{2} \cdot \cos \frac{\phi}{2} \cdot\left[K_{I} \cdot(1+\cos \phi)-3 \cdot K_{I I} \cdot \operatorname{sen} \phi\right] \\
& K_{M M I I}=\frac{1}{2} \cdot \cos \frac{\phi}{2} \cdot\left[K_{I} \cdot \operatorname{sen} \phi+K_{I I} \cdot(3 \cdot \cos \phi-1)\right]
\end{aligned}
$$

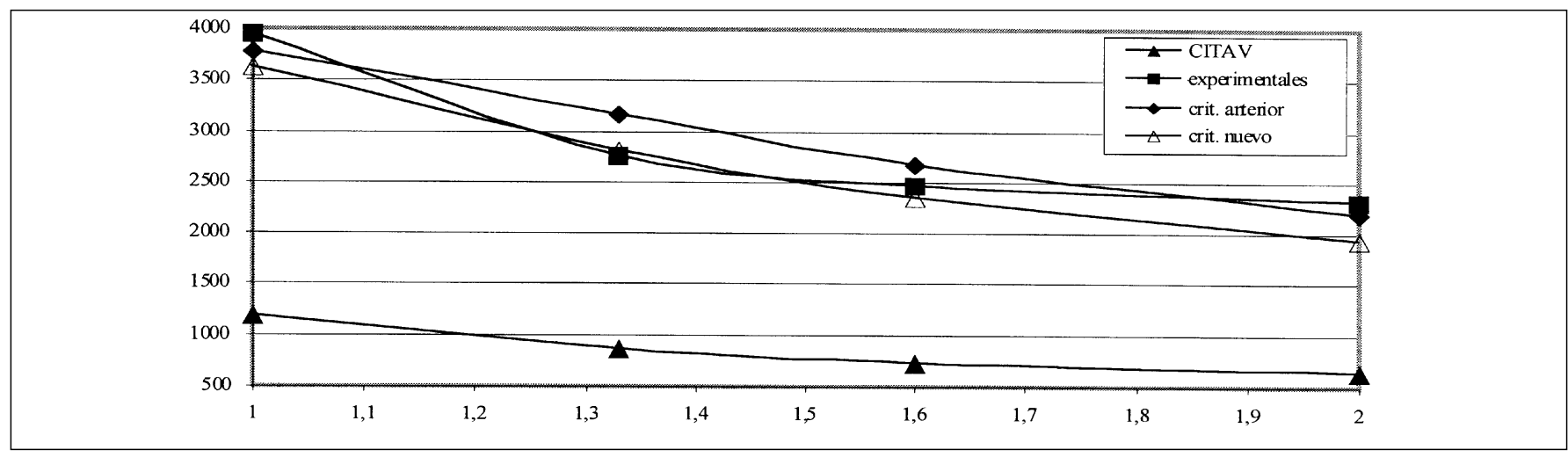

Fig. 6. Cargas de rotura en Pa para probabilidad del 5\% y cargas del CITAV. 
que dependen del ángulo $\phi$, bajo el que progresa la fisura, en relación con la dirección de la fisura original y de los factores de intensidad de tensiones convencionales en modos puros I y II. Las expresiones (10) y (11) coinciden con las propuestas por Lo et al. [11] para la definición de un modelo unificado de Mecánica de Fractura, y con las de Nuismer [12]. Estos nuevos factores de intensidad de tensión dependen de las dos componentes de tensión $\left(\sigma_{n^{\prime}} \tau\right)$ que actúan sobre la fisura.

La tasa de liberación de energía de una fisura bajo modo mixto de carga, $\mathrm{G}_{\mathrm{MM}^{\prime}}$ que considera el campo de compresión del codo se obtiene a partir de la expresión (12), donde $G_{M M I}$ y $\mathrm{G}_{\mathrm{MMII}}$ son las tasas de liberación de energía correspondientes a las porciones de modo I y II, respectivamente, y $\beta$ viene dado por la expresión (13) [10].

$$
\mathrm{G}_{\mathrm{MM}}=\left[\mathrm{G}_{\mathrm{MMI}}+\mathrm{G}_{\mathrm{MMII}}\right] \cdot[1+\beta]
$$

$$
\beta=-0,25 .\left(\frac{K_{M M I I}}{K_{M M I}+K_{M M I I}}\right)^{2.5}
$$

Expresando la tasa de liberación de energía en modo mixto, $\mathrm{G}_{\mathrm{MM}^{\prime}}$ en función del tamaño del defecto y del estado tensional es posible predecir el ángulo según el cual va a crecer la fisura en función de la carga aplicada $\left(\sigma_{n^{\prime}} \tau\right)$, sin más que buscar el ángulo crítico, $\phi_{\mathrm{c}^{\prime}}$ que hace máxima la expresión de $\mathrm{G}_{\mathrm{MM}}$.

Siempre que las grietas no tengan una elevada plasticidad o zonas de no-linealidad, se acepta que $\mathrm{K}_{\text {IIc }}$ puede referirse a $\mathrm{K}_{\mathrm{Ic}^{\prime}}$ que es una propiedad intrínseca del material, lo que equivale a decir que la tasa de liberación de energía crítica bajo carga combinada es igual a la tasa de liberación de energía crítica para modo I en el mismo material [13]. Por tanto, se supone que la tasa de liberación de energía crítica en modo mixto responde a la expresión:

$$
\mathrm{G}_{\mathrm{MMcr}}=\mathrm{K}_{\mathrm{Ic}}^{2}\left(1-v^{2}\right) / \mathrm{E}
$$

Para cada posible orientación del defecto se calcularán las tensiones normal y tangencial referidas a su plano, $\left(\sigma_{n i}, \tau_{\mathrm{i}}\right)$. Conocidas estas tensiones es posible obtener el ángulo, $\phi_{i^{\prime}}$ según el cual debería producirse la ramificación la fisura para que la tasa de liberación de energía fuese máxima. Por último, de la igualdad (15) se deriva el tamaño máximo de defecto que originó la rotura.

$$
\mathrm{G}_{\mathrm{MM}}=\mathrm{G}_{\mathrm{MM}, \mathrm{cr}}
$$

\section{CONTRASTE EXPERIMENTAL}

Por el momento y puesto que no hay experimentación en parabrisas, se irán contrastando las modificaciones a partir de los resultados de campañas anteriores desarrolladas en vidrio plano. Los resultados para placas de acristalamiento obtenidos experimentalmente, en el marco de la tesis doctoral realizada por uno de los autores [5], se comparan ahora con el nuevo criterio aquí planteado.

Para las placas de vidrio ensayadas se midió una tenacidad a fractura de $\mathrm{K}_{\mathrm{Ic}}=0.75 \mathrm{MPa} \cdot \mathrm{m}^{1 / 2}$, y se dedujo una f.d.d. de defectos superficiales, dada por una ley de Gumbel con parámetros diferentes para cada una de las cinco orientaciones ensayadas [2].

Con el fin de comprobar la validez del nuevo criterio, se utilizaron los resultados recogidos en la Tabla I de los ensayos de rotura de placas de acristalamiento para las cuatro dimensiones siguientes:

$\begin{array}{ll}\text { Placa 1: } & 2000 \times 2000 \times 5.9 \mathrm{~mm}^{3} \\ \text { Placa 2: } & 2000 \times 1600 \times 5.9 \mathrm{~mm}^{3} \\ \text { Placa 3: } & 2000 \times 1330 \times 5.9 \mathrm{~mm}^{3} \\ \text { Placa 4: } & 2000 \times 1000 \times 5.9 \mathrm{~mm}^{3}\end{array}$

llevados a cabo por el Centro de Investigación y Desarrollo de Cristalería Española, S.A., en cuya ejecución se utilizó un bastidor, preparado al efecto, en el que se probaban dos pla-

TABLA I.

CARGAS EXPERIMENTALES DE ROTURA EN PLACAS [Pa].

\begin{tabular}{|cccc|}
\hline Placa1 & Placa2 & Placa3 & Placa4 \\
\hline 2136 & 2264 & 2401 & 3861 \\
\hline 2401 & 2528 & 3734 & 4528 \\
\hline 2930 & 2930 & 3734 & 5067 \\
\hline 3067 & 3067 & 3734 & 5194 \\
\hline 3067 & 3195 & 3734 & 5331 \\
\hline 3067 & 3195 & 3734 & 5998 \\
\hline 3195 & 3195 & 4400 & 6399 \\
\hline 3195 & 3332 & 4528 & 6527 \\
\hline 3195 & 3332 & 5331 & \\
\hline 3734 & 3469 & & \\
\hline
\end{tabular}

TABLA II.

PARÁmetros DE LA F.D.D. DE Weibull PARA TODAS las CARGAS DE ROTURA EXPERIMENTALES.

\begin{tabular}{|cccccc|}
\hline & $\begin{array}{c}\text { Muestra } \\
\text { total }\end{array}$ & Placa 1 & Placa 2 & Placa 3 & Placa 4 \\
\hline$\lambda$ & -2.10 & 2067.71 & 2251.77 & 2253.61 & 3434.87 \\
\hline$\delta$ & 2.314 & 1028.15 & 880.42 & 1842.49 & 2124.93 \\
\hline$\beta$ & 2.077 & 2.077 & 2.077 & 2.077 & 2.077 \\
\hline
\end{tabular}

TABLA III.

CARGAS DE ROtURA DE LAS PlacAs ENSAYADAs[Pa]. $\mathrm{R}_{1}$ SON RESUltados EXPERIMENTALES, $R_{2}$ TEÓRICOS CON EL CRITERIO ANTERIOR, $Y R_{3}$ LOS DEL NUEVO CRITERIO DE ROTURA.

\begin{tabular}{|l|lll|lll|lll|}
\hline & \multicolumn{3}{|c|}{$\mathbf{1}_{\mathbf{0} 00}$} & \multicolumn{3}{|c|}{$\mathbf{5 \%}$} & \multicolumn{3}{|c|}{$\mathbf{5 0} \%$} \\
\cline { 2 - 10 } & $\mathbf{R}_{\mathbf{1}}$ & $\mathbf{R}_{\mathbf{2}}$ & $\mathbf{R}_{\mathbf{3}}$ & $\mathbf{R}_{\mathbf{1}}$ & $\mathbf{R}_{\mathbf{2}}$ & $\mathbf{R}_{\mathbf{3}}$ & $\mathbf{R}_{\mathbf{1}}$ & $\mathbf{R}_{\mathbf{2}}$ & $\mathbf{R}_{\mathbf{3}}$ \\
\hline Placa 1 & 2104 & 1698 & 1501 & 2313 & 2188 & 1931 & 2929 & 2793 & 2467 \\
\hline Placa 2 & 2283 & 2072 & 1830 & 2462 & $\mathbf{2 6 6 6}$ & 2351 & 2990 & 3392 & $\mathbf{2 9 9 9}$ \\
\hline Placa 3 & 2320 & $\mathbf{2 4 6 8}$ & 2177 & 2735 & $\mathbf{3 1 6 9}$ & $\mathbf{2 8 0 0}$ & 3798 & $\mathbf{4 0 1 3}$ & 3563 \\
\hline Placa 4 & 3511 & 2889 & 2798 & 3943 & 3778 & 3626 & 5216 & 4757 & 4528 \\
\hline
\end{tabular}

TABLA IV.

COEFICIENTES DE SEGURIDAD DEL CITAV Y LOS MODELOS TEÓRICOS RESPECTO A LOS RESULTADOS EXPERIMENTALES.

\begin{tabular}{|lccccc|}
\hline & Placa 1 & Placa 2 & Placa 3 & Placa 4 & Var \\
\hline CITAV & 4.80 & 4.44 & 4.34 & 4.46 & 0.039 \\
C.anterior & 1.40 & 1.20 & 1.18 & 1.39 & 0.015 \\
C.nuevo & 1.58 & 1.36 & 1.33 & 1.45 & 0.013 \\
\hline
\end{tabular}


cas simultáneamente, sometiéndolas a una presión neumática lateral uniforme cuya magnitud en el momento de la rotura queda registrada.

Puesto que hay pocos datos para cada tipo de placa, se han normalizado los resultados de todos los ensayos, comprobando que se ajustan a una f.d.d. de Weibull para mínimos (16), cuyos parámetros se han obtenido mediante el programa Extremes [8];

$$
\mathrm{F}(\mathrm{P})=1-\exp \left\{-\left(\frac{P-\lambda}{\delta}\right)^{\beta}\right\}
$$

La figura 5 recoge en papel probabilístico de Weibull para mínimos los resultados de rotura de los ensayos.

A partir de los parámetros obtenidos para la muestra normalizada se han calculado los parámetros para cada una de las cuatro poblaciones de cargas de rotura (Tabla II).

En la Tabla III se incluyen los resultados de las cargas de rotura correspondientes a los ensayos, ya tratados estadísticamente, así como los resultados predichos para el dimensionamiento de placas según el criterio anterior y el criterio nuevo.

Como probabilidades de rotura se han elegido $1 \%, 5 \%$ y $50 \%$ por considerar que son las más significativas. Aunque, en principio, por similitud con otros materiales constructivos, podría adoptarse la probabilidad de rotura del 5\% como característica para el dimensionamiento, las características de fragilidad del vidrio, el elevado número de placas de acristalamiento en un edificio alto, unido a las graves repercusiones que conlleva el fallo de este tipo de elementos, parece más aconsejable considerar aquí la probabilidad de rotura del $1 \%$ como más representativa.

En la figura 6 se representan, a modo de comparación, los valores de los resultados de las cargas de dimensionamiento para las cuatro placas, según las tablas de valores editadas por el CITAV [14], junto a los resultados experimentales y a los resultados teóricos, para un 5\% de probabilidad de rotura, predichos según los dos criterios, anterior y actual.

Por último, para las cargas de roturas obtenidas mediante los criterios teóricos y mediante las tablas del CITAV [14] se han obtenido los coeficientes de seguridad referidos a los resultados experimentales de placas anteriormente comentados (ver Tabla IV).

Para los casos de placas aquí presentados el método del CITAV y los criterios anterior y nuevo son muy buenos. El criterio nuevo mejora moderadamente al anterior.

Los criterios probabilísticos se adaptarían sin problemas a un problema diferente, y además con ellos es posible conocer el coeficiente de seguridad real, es decir, la probabilidad de rotura a la que están asociados.

El modelo de dimensionamiento para placas de vidrio ha de ser modificado para que sea aplicable al dimensionamiento de elementos de vidrio laminar curvo, concretamente a parabrisas. Esto supone un salto cualitativo importante, pues no sólo cambian la geometría y las condiciones de contorno sino también el material.

\section{CONSIDERACIONES FINALES}

1) Con las mejoras introducidas se espera que, tanto los ensayos de flexión a cuatro puntos para la determinación de la f.d.d. de defectos como el contraste de los valores de parámetros de fractura por indentación mediante ensayos de fle- xión con muestras indentadas impliquen al menos pequeñas variaciones cuantitativas, que contribuirán a un mejor ajuste, con la consiguiente reducción en la dispersión de las diferencias entre los resultados predichos y los resultados experimentales.

2) El criterio de fractura empleado en el modelo de dimensionamiento de placas de vidrio adolece aún de ciertas deficiencias que deben ser subsanadas para alcanzar una mayor fiabilidad en la predicción de rotura. Una posible mejora consiste en incluir en el balance energético que determina la propagación de la fisura la pérdida de energía elástica disponible debido al codo que se forma entre la fisura original y la ramificación.

3) Como parámetro crítico $\left(\mathrm{G}_{\mathrm{MM}, \mathrm{cr}}\right)$ o magnitud de comparación de la resistencia del material en la rotura bajo modo mixto se adopta la correspondiente al crecimiento de la fisura en modo I.

4) El nuevo criterio permite una correcta predicción de la carga de rotura, según se comprueba experimentalmente, proporcionando un coeficiente de seguridad prácticamente constante en el rango de placas estudiado.

5) La adopción de una probabilidad del $1 \%$ como valor característico, o de referencia, parece adecuada, o incluso necesaria en el dimensionamiento de estructuras de vidrio. Ello implica, sin embargo, una determinación más fiable de los parámetros del modelo, con la consiguiente necesidad de un mayor volumen de experimentación.

\section{AGRADECIMIENTOS}

Los autores desean expresar su agradecimiento, tanto a la Universidad de Oviedo por la financiación recibida a través del proyecto NP-98-524-1, como al Centro de Investigación y Desarrollo y a SEKURIT, ambas pertenecientes a Cristalería Española, S.A. por el asesoramiento y la ayuda financiera prestados en el desarrollo de los estudios aquí presentados.

\section{BIBLIOGRAFÍA}

1. I. Viña Olay "Análisis no-lineal de rotura de placas planas de vidrio con consideración de criterios probabilísticos", Universidad de Oviedo. Tesis Doctoral, 1987.

2. A. Fernández Canteli, I. Viña Olay, A. Bernardo Sánchez, "Dimensionamiento de placas de acristalamiento para edificios mediante un modelo probabilístico de rotura", Materiales de construcción, vol. 46, n'242-243, 1996

3. A. Pajares, F. Guiberteau, F.L. Cumbrera, A. Dominguez-Rodriguez, “Un modelo para explicar la configuración de fisuras generadas mediante ensayos Vickers a diferentes cargas en 4Y-PSZ", Anales de Mecánica de la Fractura, Vol. 9, pag 22-29, 1992.

4. D. Casellas, L. Llanes, M. Anglada, "Influencia de la microestructura en la fractura de Y-TZP", Anales de Mecánica de la Fractura, Vol. 15, 1998.

5. A. Bernardo Sánchez, "Cálculo probabilístico en rotura de acristalamientos mediante utilización de parámetros de fractura", Universidad de Oviedo. Tesis Doctoral, 1994.

6. J.M. Fernández Navarro, "El vidrio". Consejo Superior de Investigaciones Científicas. Instituto de Cerámica y Vidrio. 1991

7. Engineered Materials Handbook. "Ceramics and Glasses" ASM, 1991.

8. E. Castillo, E. Alvarez, A. Cobo, T. Herrero, "An expert system for the analysis of extreme value problems", Universidad de Cantabria, 1993.

9. R. Podleschny, "Untersuchungen zum Instabilitätsverhalten scherbeanspruchter Risse". Tesis doctoral. Bochum, 1993.

10. R. Podleschny, J.F. Kalthoff, "A novel mode-II fracture criterion", $10^{\text {th }}$ Biennial European Conference on Fracture. Berlin, 1994.

11. K.W. Lo, T. Tamilselvan, K.-H. Chau, M.M. Zhao, "A unified model for fracture mechanics". Eng. Fracture Mechanics, Vol.54, No2, pag.189-210, 1996.

12. R.J. Nuismer, "An energy release rate criterion for mixed mode fracture", International Journal of Fracture, vol 11, N $\mathrm{N}^{\mathrm{a}}$ 2, Abril 1975.

13. M.A. Hussain, S.L. Pu, J. Underwood, "Strain energy release rate for a crack under combined mode I and mode II". Fracture Analysis, ASTM STP 560, pag. 2- 28, 1974

14. Manual del vidrio. CITAV, 1996 